\title{
Similar IgE Binding Patterns in Gulf of Mexico and Southeast Asian Shrimp Species in US Shrimp Allergic Patients
}

\author{
Sara Anvari ${ }^{1}$, Shea Brunner ${ }^{1}$, Karen Tuano $\mathrm{S}^{1}$, Brenda Bin $\mathrm{Su}^{1}$, Shaymaviswanathan \\ Karnaneedi $^{2}$, Andreas Lopata ${ }^{2}$, and Carla Davis ${ }^{1}$ \\ ${ }^{1}$ Baylor College of Medicine Department of Pediatrics \\ ${ }^{2}$ James Cook University Australian Institute of Tropical Health and Medicine
}

February 9, 2022

Similar IgE Binding Patterns in Gulf of Mexico and Southeast Asian Shrimp Species in US Shrimp Allergic Patients

Sara Anvari ${ }^{1,2 *}$, Shea Brunner ${ }^{1,2 *}$, Karen Tuano ${ }^{1,2}$, Brenda Bin $\mathrm{Su}^{1,2}$, Shaymaviswanathan Karnaneed ${ }^{3}$, Andreas L. Lopata ${ }^{3}$, Carla M. Davis ${ }^{1,2}$

${ }^{1}$ Baylor College of Medicine, Texas Children's Hospital, Department of Pediatrics, Section of Immunology, Allergy and Retrovirology, Houston, Texas

${ }^{2}$ Baylor College of Medicine, William T. Shearer Center for Human Immunobiology, Houston, Texas

${ }^{3}$ James Cook University, Australian Institute of Tropical Health and Medicine, Centre for Molecular Therapeutics, Douglas, QLD, Australia

*co-first authors

Corresponding Author:

Carla M. Davis

1102 Bates Ave, Ste 330

Houston, TX 77030

Phone 832-824-1319

Fax 832-825-1260

Funding Source : The project was supported by the NIH-NIAID grant 1R34AI57948-01

Total Word Count : 599

Capsule Summary :

Key Words : Shrimp allergy, Shrimp oral immunotherapy, tropomyosin, anaphylaxis, food allergy,

Abbreviations: SA-Shrimp allergy, sIgE-specific IgE,Dermatophagoides pteronyssinus ( Der p1) ,Dermatophagoides farinae ( Der f1), recombinantPenaeus aztecus ( rPen a), recombinant Penaeus monodon ( rPen $\mathrm{m}$ )

\section{Conflict of Interest Statement:}


Drs. Anvari and Davis receive research contract funding from DBV Technologies, Regeneron and Aimmune Therapeutics. Dr. Davis is a consultant for Moonlight Therapeutics. The other authors declare that they have no known competing financial interests or personal relationships that could have appeared to influence the work reported in this paper.

\section{Abstract}

Background: Shrimp allergy is a leading cause of anaphylaxis in patients with food allergies and the most common cause of food allergy in adults. There are known conserved B-cell allergen IgE binding epitopes that would predict cross-reactivity of shellfish allergens, but patient shrimp specific IgE binding has not been assessed in different shrimp species.

Objective: This study examines the IgE binding pattern to major shrimp proteins in two different shrimp species among US shrimp allergic patients.

Methods: Patients with a physician-diagnosed shrimp allergy were recruited for this analysis. Skin prick testing to raw shrimp, cooked shrimp, shrimp extract, Dermatophagoides pteronyssinus (Der p1), Dermatophagoides farinae (Der f1), cockroach, codfish, crab, lobster, and oyster extracts were performed in all patients. Serum collected from US shrimp allergic patients were analyzed by Western Blot to assess the IgE binding patterns for major shrimp allergens in two different shrimp species, Penaeus aztecus (Brown shrimp) and Penaeus monodon (Black tiger shrimp).

Results : Specific $\operatorname{IgE}$ ( $\mathrm{sIgE}$ ) binding to the major and minor shrimp allergens including tropomyosin, arginine kinase, myosin light chain, hemocyanin and sarcoplasmic calcium binding protein was evaluated. The sIgE binding pattern in US shrimp allergic patients for the major heat-stable shrimp allergens tropomyosin, myosin light chain and sarcoplasmic protein were similar for different shrimp speciesPenaeus aztecus (Brown shrimp; Gulf of Mexico) and Penaeus monodon (Black tiger shrimp; Southeast Asia), suggesting conservation of epitopes between species.

Conclusion : Given similar sIgE binding patterns for the major shrimp proteins in the two different shrimp species, shared epitopes are observed between shrimp species. This knowledge may help with the diagnosis of shrimp allergy and cross-reactivity, as it could lend itself to the development of a component-resolved diagnostic assay to identify sIgE to epitopes in different shrimp species.

\section{To the Editor:}

Shellfish allergy (SA) is a leading cause of food-induced anaphylaxis ${ }^{1}$ and the most common cause of adultonset food allergy, with 1-3\% of the United States (US) population affected. ${ }^{2-4}$. Nearly half (45\%) of US adults with SA report utilizing emergency services for SA symptoms over their lifetime, ${ }^{2}$ remaining at-risk for lethal allergic reactions. Several allergenic proteins have been identified across shellfish species, including tropomyosin (TM), arginine kinase (AK), myosin light chain (MLC), sarcoplasmic calcium binding protein (SCP), hemocyanin, troponin $\mathrm{C}$, and triosephosphate isomerase. ${ }^{5}$ The allergens of major importance in SA are the muscle proteins TM and AK. TM, the major allergen with specific-IgE antibodies in $<90 \%$ of SA patients, is associated with severe clinical reactivity. AK is a pan-allergen with cross-reactivity with crustaceans and cephalopods. ${ }^{5}$

Cross-reactivity has been observed clinically when SA patients ingest various invertebrate species with subsequent allergic reactions, but further study of shrimp sIgE-binding between different shrimp species is needed. ${ }^{6,7}$ This study examined the sIgE-binding patterns to 2 shrimp species from the Gulf of Mexico and Southeast Asia in US SA patients.

SA patients with shrimp-induced allergic reactions and positive immediate skin prick testing (IHST) and/or shrimp sIgE ImmunoCAP levels were recruited from the Baylor College of Medicine (BCM) Allergy and Immunology Clinics. The study was approved by the BCM IRB and all participants provided written, informed consent. The patients underwent IHST to shrimp extract (mixture ofPenaeus borealis, Penaeus monodon, Metapenaeus barbata and Metapenaeopsis joyner), raw shrimp, cooked shrimp,Dermatophagoides 
pteronyssinus (Der p1, Der p 10) ,Dermatophagoides farinae (Der f1), cockroach, codfish, crab, lobster, and oyster using extracts from Greer. ImmunoCAP ISAC testing (ThermoFisher) assessed total IgE as well as sIgE levels for shrimp, recombinant Penaeus aztecus (TM), Der p10, Der p1, Der p2, recombinant Penaeus monodonAK, MLC, SCP, troponin C, crab, lobster, cockroach, clam, and oyster. Western blot (WB) analysis of sIgE-binding profile to the major allergenic proteins TM, AK, MLC, and SCP in Penaeus aztecus(Brown shrimp) and $P$ monodon (Black tiger shrimp) was performed.

Fifteen SA patients between the ages of 2-17 years were enrolled.(Table 1) The most frequent symptoms were urticaria and angioedema. Forty-percent had antihistamine treatment, one required steroids, and none required epinephrine or albuterol. When examining sIgE-binding patterns to the major allergens, WB revealed nearly identical sIgE-binding profiles to tropomyosin, MLC and SCP in both species in the heated shrimp extracts.(Figure 1.) Eight subjects (53\%) demonstrated sIgE-binding to TM and six to SCP (40\%). There was variability in the binding to other shrimp proteins, particularly in the unheated shrimp. ${ }^{5}$ The proposed sIgE-binding allergens have been identified by molecular weight, ${ }^{5,6}$ but need to be confirmed in future molecular studies.

The presence of Southeast Asian Black tiger shrimp sIgE has been established and quantified through ImmunoCAPISAC testing, with over $50 \%$ of patients having binding to TM, $33 \%$ to SCP, $27 \%$ to AK and $13 \%$ each to MLC and troponin C. ${ }^{6}$ Nevertheless, each individual patient's binding pattern in our study was similar between the two species, both unheated (Fig 1A,B) and heated proteins (Fig 1C,D). These similar sIgE-binding patterns to the major allergens suggested conservation of the epitopes between different species of shrimp.

According to the Food and Agricultural Organization of the United Nations, Penaeus aztecus is native to the north-western Atlantic Ocean and Gulf of Mexico, while Penaeus monodon is native to the coasts of Southeast Asia, South Asia, East Africa, and Australia. Our study demonstrates shared epitopes among the major shrimp proteins for Gulf Brown shrimp and Southeast Asian Black tiger shrimp in US shrimp allergic subjects, implicating shared diagnostic and therapeutic potential for SA to varied shrimp species.

\section{References}

1. Conrado AB, Patel N, Turner PJ. Global patterns in anaphylaxis due to specific foods: A systematic review. J Allergy Clin Immunol. 2021 May 1;S0091-6749(21)00665-5.

2. Warren CM, Aktas OZ, Gupta RS, Davis CM. Prevalence and characteristics of adult shellfish allergy in the United States. J Allergy Clin Immunol. 2019 Nov; 144(5):1435-1438.

3. Wang HT, Warren CM, Gupta RS, Davis CM. Prevalence and characteristic of shellfish allergy in the pediatric population of the United States. J Allergy Clin Immunol Pract. 2020 Apr; 8(4):1359-1370.

4. Wood RA. Food allergen immunotherapy: Current status and prospects for the future. J Allergy Clin Immunol. 2016 Apr;137(4):973-982.

5. Ruethers T, Taki AC, Johnston EB, Nugraha R, Le TTK, Kalic T, McLean TR, Kamath SD, Lopata AL. Seafood allergy: A comprehensive review of fish and shellfish allergens. Mol Immunol. 2018 Aug;100:28-57. doi: 10.1016/j.molimm.2018.04.008. Epub 2018 May 30. PMID: 29858102..

6. Nugraha, R., Kamath, S., Johnston, E., Karnaneedi, S., Ruethers, T. and Lopata, A., 2019. Conservation Analysis of B-Cell Allergen Epitopes to Predict Clinical Cross-Reactivity Between Shellfish and Inhalant Invertebrate Allergens. Frontiers in Immunology, 10.

7. MacGinnite A. Update on Potential Therapies for IgE-Mediated Food Allergy. Curr Allergy asthma Rep. 2017 Jan;17(1):4.

\section{Figure 1 Legend:}

Figure 1 A, B, C, D: Western blot analysis of the major and minor shrimp allergen proteins for different shrimp species in shrimp allergic and healthy controls (A) Penaeus aztecus (Brown shrimp), (B) Penaeus monodon (Black tiger shrimp), (C) heated extract of Penaeus aztecus (Brown shrimp), (D) heated extract ofPenaeus monodon (Black tiger shrimp). The location of sIgE to proposed allergens are highlighted with red boxes. HC: healthy control. 


\section{Hosted file}

Figure 1_Submitted_2_1_22.docx available at https://authorea.com/users/459664/articles/ 555864-similar-ige-binding-patterns-in-gulf-of-mexico-and-southeast-asian-shrimpspecies-in-us-shrimp-allergic-patients

\section{Hosted file}

Table I_Submitted_Allergy_2_6_22.docx available at https://authorea.com/users/459664/ articles/555864-similar-ige-binding-patterns-in-gulf-of-mexico-and-southeast-asianshrimp-species-in-us-shrimp-allergic-patients 